ARTÍCULO

\title{
Aspectos pedagógicos del conectivismo y su relación con redes sociales y ecologías del aprendizaje
}

\author{
Jaime Andrés Torres Ortiz' \\ Thiago Henrique Barnabé Corrêa"

\section{RESUMEN}

Esta investigación se desarrolla a partir de la revisión sistemática de literatura, método que permitió seleccionar aquellas investigaciones que se incluyen en la pregunta: ¿Qué experiencias educativas se presentan a través del desarrollo e implementación del conectivismo, en los procesos educativos en red y su relación con las perspectivas sistémicas y ecológicas del flujo de información y conocimiento? Se escogieron investigaciones sobre conectivismo; el aprendizaje autónomo en red; las experiencias constructivistas, cognitivas, sociales y culturales en redes educativas virtuales; la dinámica conectivista en las redes de aprendizaje y comunidades de práctica. Entre las conclusiones se evidencian pedagogías emergentes enfocadas en la incidencia del conectivismo en las ecologías del aprendizaje, los ecosistemas, el actor-red, el eco-conectivismo y, el aprendizaje nómada. Entre otras pedagogías que se contextualizan en comunidades de práctica y los ecosistemas educativos.

\section{PALABRAS CLAVE}

redes de aprendizaje; cognición social; comunidades de práctica. 


\title{
PEDAGOGICAL ASPECTS OF CONNECTIVISM AND ITS RELATIONSHIP WITH SOCIAL NETWORKS AND LEARNING ECOLOGIES
}

\begin{abstract}
This research is developed from the systematic review of literature, a method that allowed to select those researches that are included in the question: What educational experiences are presented through the development and implementation of connectivism, in the educational processes in the network and its relation with the systemic and ecological perspectives of the flow of information and knowledge? Research on connectivism, online autonomous learning, constructivist; cognitive; social and cultural experiences in virtual educational networks; connectivist dynamics in learning networks and communities of practice were chosen. Among the conclusions are emerging pedagogies focused on the incidence of connectivism in the ecologies of learning, ecosystems, the actor-network, eco-connectivism, and nomadic learning. Among other pedagogies that are contextualized in communities of practice and educational ecosystems.
\end{abstract}

\section{KEYWORDS}

learning networks; social cognition; communities of practice.

\section{ASPECTOS PEDAGÓGICOS DO CONECTIVISMO E SUA RELAÇÃO COM REDES SOCIAIS E ECOLOGIAS DE APRENDIZAGEM}

\section{RESUMO}

A presente pesquisa se desenvolveu a partir de uma revisão sistemática da literatura, método que permitiu selecionar as investigações que versam as seguintes perguntas: Quais experiências educativas se apresentam por meio do desenvolvimento e implementação do conectivismo nos processos educativos em rede e qual a relação do conectivismo com as perspectivas sistêmicas e ecológicas do fluxo de informação e conhecimento? Para tal, escolheu-se investigações sobre conectivismo; aprendizagem autônoma on-line; as experiências construtivistas, cognitivas, sociais e culturais em redes educativas virtuais; a dinâmica conectivista nas redes de aprendizagem e comunidades de prática. Entre as considerações finais, evidencia-se pedagogias emergentes focadas na incidência do conectivismo nas ecologias da aprendizagem, os ecossistemas, os atores-rede, o eco-conectivismo e a aprendizagem nômade, entre outras pedagogias que se contextualizam em comunidades de prática e ecossistemas educativos.

PALAVRAS-CHAVE

redes de aprendizagem; cognição social; comunidades de prática. 


\section{INTRODUCCIÓN}

En la última década se ha hablado mucho sobre la fluidez del conocimiento donde el mundo está regido por una nueva sociedad, interconectada, en interacción continua con la información y el flujo del conocimiento. El presente trabajo de investigación, aborda a partir de una revisión sistemática de literatura un conjunto de trabajos investigativos, los cuales abordan el conectivismo y su relación con los procesos pedagógicos, el aprendizaje y las redes virtuales. Los trabajos analizados apuntan hacia los cambios ocurridos en la sociedad en la era de la cibernética, sobre todo, transformaciones de escala en el marco cognitivo (Falcão, 2006). Como trae Rodríguez y Molero (2009), la inclusión de la tecnología y la identificación de conexiones como actividades de aprendizaje, empieza a mover las teorías de aprendizaje hacia la edad digital.

En los últimos años se ha hablado mucho sobre la fluidez del conocimiento en que el mundo está regido por una nueva sociedad, interconectada y sistematizada. Superficialmente, tratamos la cultura digital como un evento aislado y puntual por sí solo, ignorando que este ambiente cultural fue construido y está definido históricamente, por lo tanto, es un producto de diversas acciones sociales que se comunican.

En este ámbito, las ideas educativas y pedagógicas de los canadienses George Siemens (2005) y Stephen Downes (2010) han emergido como parte de la discusión sobre los procesos pedagógicos en la era digital y de la información. El desarrollo y uso de internet y todo el diseño de software que este implica, se ha desarrollado a la par del trabajo de Siemens (2005), quien desplegó y fundamentó una nueva propuesta teórica para comprender el proceso del aprendizaje y las experiencias que se configuran allí, entre los procesos de enseñanza y aprendizaje.

La primera visión sobre el conectivismo nos lleva a considerar este constructo en teoría de aprendizaje de la era digital, pero también, podemos verlo en su bivalencia, o sea, como perspectiva pedagógica y de currículo que implica cambio en los paradigmas metodológicos, una vez que la matriz de ideas del conectivismo no abandona la interacción del sujeto con el Otro y su medio, sea esa interacción directa o no, trae consigo la cultura mediática, digital y convergente como componente de análisis (Corrêa, Moreno y Pineda, 2018; Santaella, 2003).

La pregunta abordada desde la metodología de revisión sistemática de literatura refiere: a ¿Qué experiencias educativas se presentan a través del desarrollo e implementación del conectivismo como parte de los procesos educativos en red y su relación con las perspectivas sistémicas y ecológicas del flujo de información y conocimiento? A partir de esta pregunta se presentan estudios que evidencian actualmente el complejo sistema educativo, cognitivo y social en las redes, el cual aporta nuevas miradas al desarrollo de la pedagogía y las ecologías del aprendizaje en un mundo digital.

\section{REFERENTES METODOLÓGICOS}

El desarrollo metodológico para la búsqueda, selección y elaboración de información se fundamenta a partir de los planteamientos metodológicos propuestos 
por el manual Cochrane (2011) sobre la revisión sistemática de literatura, a través de la cual se debe reunir o juntar toda información o documentos que sirvan de soporte para acertar con todo criterio de elegibilidad propuesto, y lograr mayor rigurosidad en la selección y análisis de información en la investigación. Posterior a esta condición se escogieron los artículos acordes y se analizaron e interpretaron cada uno de ellos con el fin de generar las temáticas más importantes a ser abordadas en su conjunto.

Se toma en cuenta los criterios de selectividad de la pregunta establecida según el procedimiento planteado por el manual Cochrane (2011), la cual se refiere a: ¿Qué experiencias educativas se presentan a través del desarrollo e implementación del conectivismo como parte de los procesos educativos en red, y su relación con las perspectivas sistémicas y ecológicas del flujo de información y conocimiento? A partir de la anterior pregunta se explican a continuación los criterios PICO (población, intervención, comparación y outcome) según el manual mencionado. Con respecto a la población; esta refiere a estudiantes y docentes de educación a distancia y virtual; en este proceso se hace una intervención situada en las experiencias educativas en educación media y educación superior que integran el uso de internet.

La base de datos a partir de la cual de obtuvieron los documentos revisados fue Web of Science. La búsqueda en los conceptos o criterios de inclusión fueron ediciones de revistas indexadas entre los últimos 5 años, entre 2013 y 2018, tomando en cuenta los cambios estructurales de los procesos educativos generados por líneas de desarrollo planteadas por la Naciones Unidas para la Educación, la Ciencia y la Cultura (UNESCO), Organización de Estados Iberoamericanos para la Educación, la Ciencia y la Cultura (OEI) y Organización de las Naciones Unidas (ONU). Se complementan conceptos que son inherentes al curso del estudio y que refieren al conectivismo en sus generalidades, las tendencias y modelos pedagógicos que aportan al fundamento teórico y redes de aprendizaje colaborativo. Consolidando el proceso metodológico, se excluyeron aquellas revistas y publicaciones que fueran de años anteriores al 2013 y, demás fuentes de consulta secundaria que no abordaran los estudios y análisis de tendencias educativas situadas en el contexto de educación superior.

Respecto de las comparaciones desarrolladas se analizan aquellas experiencias educativas suficientemente ejecutadas que hacen uso e integran el internet en su dinámica, con relación a las experiencias que evidencian el uso de internet $\mathrm{o}$, hacen poco uso de esta herramienta informática y comunicacional. En el mismo sentido, según el desarrollo de la pregunta según el Manual Cochrane de Revisiones Sistemáticas de Intervenciones se analizan los resultados obtenidos (outcome) en el marco general de las investigaciones y, se integran todos los análisis en las experiencias educativas que involucran el uso de internet y su relación con las perspectivas ecológicas, ecosistémicas y sistémicas que son transversales a los fundamentos pedagógicos del conectivismo.

Precisamente, los trabajos o investigaciones revisadas presentan los conceptos de las ecologías del aprendizaje y su relación con los principios ecosistémicos que están dados a partir de las investigaciones de Brown (1999) (apud Bozkurt et 
al., 2016), y las propuestas de Hill, Wilson y Watson (2004) sobre los principios ecológicos, y sistémicos que caracterizan un aprendizaje en interacción con otros sistemas, de orden mutualista, sostenibles en el tiempo, orientado a comunidades que comparten ideas, conceptos, conocimientos, $y$ en consonancia con el principio ecológico de la sostenibilidad y la relación directa con la condición de la naturaleza.

Los criterios de inclusión anteriormente mencionados fueron referentes para contemplar el desarrollo de la presente investigación. No obstante, a la par de estos aspectos anteriormente mencionados se tuvieron en cuenta los siguientes criterios de exclusión: artículos de investigación que comprendieran temas en profundidad con respecto al diseño y uso de Massive Online Open Courses (MOOC). Se excluyeron investigaciones que comprendieran la implementación del conectivismo en disciplinas diferentes a las áreas educativas y humanísticas y no se involucraron artículo o estudios que hagan referencia al conectivismo en su estudio y análisis del diseño y uso de software.

\section{EXPERIENCIAS EVIDENCIADAS A TRAVÉS DE LA REVISIÓN SISTEMÁTICA DE LITERATURA}

Con base en el proceso de búsqueda, selección y elaboración de información conforme los procedimientos propuestos por el Manual Cochrane de Revisiones Sistemáticas de Intervenciones, se exponen las experiencias educativas que se presentan a través del desarrollo e implementación del conectivismo como parte de los procesos educativos, y su relación con las perspectivas sistémicas y ecológicas del flujo de información y conocimiento.

Más que la visión de una base teórica, los tres subtítulos que se presentarán asumen el conectivismo como una posible forma de gestión del conocimiento (Rodríguez y Molero, 2009). En consonancia con las relaciones conceptuales, teóricas y prácticas que se evidencian durante el análisis de la información, en este sentido, las experiencias presentadas a continuación se sitúan en el campo del conectivismo con relación con el aprendizaje autónomo y en red; el conectivismo y su relación con las experiencias constructivistas, cognitivas, sociales y culturales en redes educativas virtuales y; el conectivismo en la dinámica de redes de aprendizaje y comunidades de práctica.

\section{EL CONECTIVISMO COMO EXPERIENCIA EDUCATIVASISTÉMICA Y DE APRENDIZAJE AUTÓNOMO EN RED}

Las revisiones de artículos sobre el conectivismo y análisis de esta experiencia educativa presentan elementos de interés que aportan al desarrollo del concepto y su relación con aspectos propios del aprendizaje y la enseñanza. En este sentido, se encuentran categorías educativas que dan cuenta de una integración entre procesos de enseñanza y aprendizaje inmersos en una red abierta y dinámica sustentada en internet y, un sistema abierto que se configura con los diseños tecnológicos de software y, demás ambientes virtuales y presenciales que acompañan la experiencia 
educativa, en cualquier parte del mundo, en relación con cualquier persona interesada en hacer parte de una comunidad o una red (Mabuan, 2018).

Con lo anterior, las dinámicas del aprender se integran hacia un horizonte informacional y educativo caracterizado por aprendizajes en donde se enfoca con mayor profundidad el conectivismo destacando capacidad autorreflexiva, crítica, autónoma, orientada al aprendizaje efectivo a través de capacidades autorreguladoras y motivacionales que inciden directamente en la búsqueda, selección, apropiación de nuevos conceptos para el desarrollo personal y profesional (Muni y Rabell, 2017). Por lo cual, conformar la red desde el conectivismo o, formar parte de esta, implica además, generar acciones autónomas para el aprendizaje, inmersos en un contexto sistémico en donde surgen conceptos como ecologías del aprendizaje, y ecosistemas de aprendizaje.

Atendiendo a los argumentos de AlDahdouh (2018), el aprendizaje a través de los principios del conectivismo determina una relación en red, caracterizada por la capacidad reflexiva, crítica y propositiva, y fundamentada en la iniciativa por parte del individuo para desarrollar autonomía y capacidad participativa. En este sentido, y siguiendo a Hassan (2018), las experiencias por parte del estudiante en el contexto de la red y, del uso de internet se integran a la capacidad para tomar decisiones, ejercer por cuenta propia sus acciones, integrarse a fuentes de información a partir de las cuales pueda conformar una red, desarrollar autoeficacia por medio de estrategias motivacionales que le permitan promover metas de aprendizaje y aplicar métodos instruccionales previamente asignados (Borna y Fouladchang, 2018).

Sobre la base de estas metodologías instruccionales surgen experiencias asociadas a un aprendizaje autodirigido y autónomo que se fortalece con la experiencia en la red y se apoya de tecnologías semánticas (Rodríguez et al., 2017; Vas, Weber y Gkoumas, 2018). Sobre esta base de trabajo se comprenden metodologías y tendencias que hacen parte de novedosas formas de potenciar el proceso de aprender a través de mecanismos que promueven el conocimiento e información compartida, la configuración de conceptos transversales como el aprendizaje rizomático en donde el estudiante sea actor y parte de la red y se integre a comunidades de práctica en la indagación y colaboratividad para compartir experiencias y conocimientos (Bozkurt y Keefer, 2018).

En especial, existe un aprendizaje reticular que se configura a partir de la conformación de un pensamiento jerárquico en donde existe una estructura arbórea que se organiza según las vertientes del conocimiento aprendido. Esto se fundamenta en los aporte de Deleuze y Guattari (1987) citados por Bozkurt y Keefer (2018), quienes confluyen su propuesta en los planteamientos del aprendizaje rizomático como un sistema de raíces con muchas conexiones entre sí (Le Grange, 2007 apud Bozkurt y Keefer, 2018), que se diferencia del aprendizaje arborescente porque este último; el rizomático, no es estructurado, jerárquico o totalitario; sino, interconectado, horizontal y multi-relacional, para efectos de un aprendizaje que se relaciona con muchos elementos, se formaliza en la práctica o se mantiene a nivel abstracto del pensamiento, lleno de rupturas y orientado a generar nuevas líneas conforme los viejos orígenes, y no adaptable tan fácilmente a los conceptos tradicionales o estructuras jerárquicas del conocimiento. 
Sobre los referentes anteriormente planteados se identifican tensiones continuas y recurrentes entre la dinámica de los procesos formativos, el flujo de información y del conocimiento y la manera como este es adquirido o apropiado por los aprendices, comprendiendo que la interacción continua con la información y la relación con otros individuos conlleva a una continua exposición de los sentidos que se construyen alrededor de ciertos saberes. Con todo lo anterior, un factor instrumental que incide en el conectivismo y su abordaje sistémico y ambiental son los diseños instruccionales y los métodos de seguimiento y control del proceso educativo, demostrando en ocasiones un aislamiento poco reflexivo y crítico que afecta la capacidad para generar lazos y compromisos humanos entre los actores involucrados en los procesos (Pando, 2018). De esta forma, las redes que se conforman, pueden convertirse en fríos sistemas de interrelación que no dejan de ser más que un medio dinámico de transmisión de información, generando mucho conocimiento acumulado y a la vez, mucha ignorancia por el escaso discernimiento y significación (Zapata-Ros, 2015).

Solo en la medida que las experiencias educativas a través de internet se integran en procesos complejos que configuran redes, transformando las existentes y configurando unas nuevas; se puede así mismo fortalecer el proceso de conformación de comunidades de aprendizaje. Para este caso, se presentan nuevos abordajes al concepto red, que se desarrollan desde el conectivismo, con es el aprendizaje rizomático, los ecosistemas de aprendizaje abierto y, el aprendizaje nómada (Bozkurt et al., 2016). En lo referente a los ecosistemas de aprendizaje abierto se parte de una red en la cual cada individuo comparte, negocia, intercambia, mezcla información, transforma o configura experiencias como parte de una relación dinámica compleja, y conforme los derechos de apertura, igualdad y libertad (Moreno y Macedo, 2014; Williams, Karousou y Mackness, 2011).

Lo anterior, se complementa a su vez con el aprendizaje nómada como un individuo que va en búsqueda del aprendizaje según sus intereses y se mantiene en continua valoración del fin o pertinencia del conocimiento que va adquiriendo (Bozkurt et al.,2016). Es un nómada por cuando interactúa para seleccionar, decidir, escoger o, transitar hacia nuevos escenarios en la red en los cuales pueda aprender conforme sus objetivos de fortalecimiento del conocimiento.

Los individuos que son nómadas del aprendizaje mantienen una fuerte motivación intrínseca que los orienta hacia nuevas redes y ven a estas mismas como algo dinámico y no estático, soportan la comunidad o red en un momento específico y, desde el conectivismo, promueven el flujo de información y participan de la estructura caótica de la red, haciendo de esta, una extensión cognitiva con principios orgánicos de un sistema que se desarrolla y transforma (Bozkurt et al., 2016). Con el fin de generar nuevas adaptaciones a la realidad/sistema y aportar al desarrollo de capacidades cognitivas y metacognitivas en el aprendizaje (Wang, Chen y Anderson, 2014).

Con lo expuesto previamente, el conectivismo a través de las redes educativas se configura como parte de una dinámica sistémica que es determinante para comprender las categorías y experiencias presentes en el contexto del presente estudio mediado por la revisión sistemática de literatura. Las tendencias investigativas sitúan 
los hallazgos en el marco de experiencias en la creación de MOOCs, como parte de una práctica educativa en la cual, la participación de estudiantes e individuos aprendientes es cada vez mayor. Es de anotar, que para la revisión de este estudio se encontraron investigaciones que relacionan directamente el uso de MOOCs con el conectivismo; no obstante, pocas investigaciones de este orden se enfocan en elementos conceptuales propios de los sistemas informáticos, ecosistemas, comprensión del funcionamiento y desarrollo del modelo conectivista en relación con ecologías del aprendizaje y/o ecosistemas de información.

Un aspecto común a los diseños de MOOCs comprende la apertura amplia y libre del sistema, por medio de cual se pueden generar múltiples interacciones y relaciones; todas estas, situadas en una experiencia de aprendizaje con base en un tema particular o conocimiento específico (Clark, Vealé y Watts, 2017). De estas experiencias educativas que están atravesadas por el conectivismo se desglosan trabajos realizados sobre el diseño de actividades de aprendizaje, por medio del cual se configuran diferentes arquetipos culturales para la solución de estos procesos (Kizito, 2016). Todos estos arquetipos están caracterizados por una configuración práctica de experiencias que están mediadas por la apropiación cultural de una comunidad, en donde el estudiante se involucra por iniciativa propia; el arquetipo implica por ende, la adopción tecnológica de un artefacto educativo diseñado para el aprendizaje con apoyo tecnológico; estos arquetipos se integran a las soluciones adoptadas para cumplir con los objetivos de aprendizaje, el trabajo colaborativo, diseño e implementación, uso de tecnologías libres y políticas de inclusión cultural, mediática, digital y convergente (Santaella, 2003), con material de apoyo prediseñado institucionalmente.

Comprendiendo el referente arquetípico cultural de la adopción y apropiación tecnológica, se evidencia en el campo de la investigación el aumento de experiencia educativas mediadas por el aprendizaje a través de las redes educativas (Clarà y Barberà, 2014). Esta práctica comprende un abordaje novedoso en la desescolarización que se sitúa en experiencia culturales distintas a la tradicionales en donde la relación aprendizaje y conectividad es directamente proporcional, con lo cual, los procesos de enseñanza se reconceptualizan, orientando su fin hacia la conformación y mantenimiento de redes en cuanto comunidades de práctica.

Desde esta perspectiva de configuración de prácticas culturales situadas en el marco conceptual del conectivismo, se identifican diferentes procesos educativos y sistémicos que mantienen en el plano teórico y práctico, una fuerte relación con las acciones de enseñanza y aprendizaje aunadas al referente cognitivo, como extensión motivacional que se encadena al flujo de la información por vía internet. Según Trnova y Trna (2015), los procesos motivacionales se encadenan en red, a partir de la actividad autónoma en interacción con la información presente en el sistema, el flujo o recorrido que esta presenta se condensa en nodos de mayor compilación de información por medio de los cuales de organiza, se transforma, se mezcla o se combina y, se transfiere hacia otros flujos de la red, correspondiendo con una ecología del aprendizaje que mantiene una lógica conectivista para promover conocimientos y comunidades con identidad cultural (Bozkurt y Keefer, 2018). 
Los anteriores planteamientos se integran a referentes propios de la autonomía y autoeficacia del estudiante e individuo en su capacidad para significar el aprendizaje e integrar o hacer parte de las comunidades y fortalecer experiencias significativas configurando como actor-red, los nodos de conocimiento e investigación. En este aspecto, referentes cognitivos y motivacionales propuestos por Thota y Negreiros (2015), argumentan la necesidad de trabajar en el desarrollo de la metacognición y estrategias motivacionales con el fin de generar actos reflexivos, activos y críticos, caracterizado por el refuerzo continuo de actividades de aprendizaje; en particular, el diseño de artefactos digitales que orientan y refuerzan el proceso este proceso, se comparten y se generan interacciones a través de procesos metacognitivos y de dominio afectivo para regular el aprendizaje y obtener logros.

El contexto educativo del conectivismo y su relación con el aprendizaje a través de redes educativas, comprende además, procesos motivacionales, cognitivos, y sociales culturales que determinan la forma como el individuo se integra a las redes en un continuo psicológico y afectivo por el aprender. De esta manera, las investigaciones analizadas en este primer punto presentan elementos conceptuales asociados a principios sociales sistémicos, ecosistémicos, rizomáticos y ecológicos que se pueden enmarcar en la educación ambiental como parte de una formación que desde los principios del conectivismo se constituye en referente conceptual para comprender la realidad actual en torno a los procesos de construcción del conocimiento.

De esta forma, los planteamientos complementarios propuestos por Baggaley (2012), demuestran que el conectivismo como parte de un modelo de interacción $\mathrm{y}$ relación entre individuos, comunidad y conocimiento, refiere a una disposición que asume el individuo para mantenerse conectado y actuar en la red. Lo contrario según Baggaley (2012), refiere a la desconexión, el aislamiento, la desolación o, el distanciamiento. Como quiera que el principio etimológico del término según Baggaley (2012), significa estar conectado a una red y/o comunidad, esta condición implica de manera clara y directa, la construcción de conocimiento, su flujo continuo en la red, los procesos sociales, neuronales, dinámicos, cambiantes, diversos en información y perspectivas socio-culturales que buscan superar continuamente la condición de caos y la comunicación a través de lenguajes diversos.

\section{EL CONECTIVISMO Y SU RELACIÓN CON LAS EXPERIENCIAS CONSTRUCTIVISTAS, COGNITIVAS, SOCIALES $Y$ CULTURALES EN REDES EDUCATIVAS VIRTUALES}

El conectivismo no surge para sustituir las teorías del aprendizaje, sino para reafirmar un elemento conceptual que refiere las revolución de las tecnologías digitales de la información y comunicación (TDIC), asociadas al ámbito de las discusiones sobre cómo la mente en el aprendizaje, en el ámbito de los proceso cognitivos del pensamiento, la cognición social, la información en red y, el comportamientos de los sistemas, se relacionan con el conocimiento y, por consiguiente, con el aprendizaje y enseñanza. 
En esta perspectiva, el conectivismo se convierte en una lente distinta para intentar entender el diseño (design) del proceso de enseñanza y aprendizaje mediado por la tecnología que, en clase, ha modificado la dinámica del quehacer pedagógico, maximizando las experiencias interpersonales, sobre todo, para la forma como se maneja la información y se le da significado. Esta condición remite a la necesidad de contemplar las corrientes, modelos, esquemas y demás tendencias pedagógicas que son fundamento para el sentido pedagógico del conectivismo.

Siguiendo a Zhou (2018), se puede establecer que el conectivismo y constructivismo integran nociones pedagógicas asociadas a la manera como se enseña y se aprende, en el marco del uso de las TDIC y, el internet. Toda la infraestructura digital y tecnológica que actualmente soporta las redes educativas virtuales, se sitúan en la práctica, en la interacción e intercambio de experiencias que parte de procesos constructivistas propios del individuo en donde se integra, capacidad para transmitirlas, demostrar actitudes positivas de aceptación y adopción de las tecnologías para el aprendizaje, y la habilidad para saber documentar la experiencia, escribirla y argumentarla. Esta condición requiere una acción razonada previa (basada en la teoría de la acción razonada) que implica estudio de los procesos cognitivos, motivacionales, afectivos y de la conducta expresa (Hassan, 2018). Con lo cual, el aprendizaje explicado a través del conectivismo se refleja a través de la compilación de experiencias escritas que se alojan como información en nodos, acompañadas del caos que pueda derivar de todo ese cúmulo de información, y la capacidad del estudiante para organizar sus aportes, sus saberes previos y aprendizajes significados, cuando está inmerso en una red educativa en escenarios virtuales de discusión abierta.

El constructivismo ha incidido directamente en la explicación pedagógica del conectivismo sociocultural, en la práctica, esta corriente pedagógica según Mattar (2018), establece relaciones entre estas corrientes pedagógicas que se fortalecen al configurarse el constructivismo en una cobertura o eje epistemológico que aporta elementos referentes al carácter del aprendizaje situado, activo, auténtico, experiencial y ampliado. Esto se explica a su vez, a través de la característica referente al conocimiento distribuido, auténtico, experiencial, basado en la zona de desarrollo próximo como referente conceptual por medio del cual se propone que el individuo pueda resolver los problemas de aprendizaje a través de los conocimientos que existen en la red, los aprendizajes distribuidos en las comunidades de prácticas o, los apoyos que se reciben por orientación directa de docentes o, pares y compañeros experimentados que pueden aportar en alcanzar los objetivos de aprendizaje establecidos.

A partir de lo anterior, la perspectiva constructivista según Benedetti (2018) complementa esta relación con el conectivismo al procurar balances pedagógicos y didácticos adecuados en donde la autonomía del estudiante y su relación con la comunidad e información existente en los nodos para construir y fortalecer experiencias que sean significativas; es decir, que permita crear nuevas estructuras de pensamiento, acciones prácticas y conocimientos por medio de los cuales se pueda participar y transformar las redes. 
En este sentido, las acciones colaborativas para el aprendizaje permiten alcanzar los fines referentes a las conexiones que se crean y transforman (Joksimović et al., 2018), cada una de las cuales se pueden fortalecer con el desarrollo del lenguaje y el discurso. Siendo este último, una característica muy común en las redes educativas, y para el cual se requiere un mayor énfasis en la orientación de la enseñanza y aprendizaje, con el propósito de generar lasos de trabajo entre estudiantes y un mayor nivel de acumulación de capital social para el individuo en relación con ambientes de aprendizajes distribuidos.

En el marco conceptual del constructivismo se hace presente a su vez, la corriente pedagógica cognitiva y su construcción sociocultural (Bolívar y Dávila, 2016). A partir de la cual se analiza el procesamiento de información a nivel mental y psicológico motivacional, y su distribución según los parámetros educativos propuestos por el conectivismo. Según Bolívar y Dávila (2016), la distribución de información y, el conocimiento a través de la red, está mediado por la actividad docente en la cual, este es un mediador en el desarrollo e implementación de la instruccionalidad. Sitúan la didáctica en estos contextos sistémicos como una práctica instruccional por la cual se desarrollan entrenamientos continuos que trabaje instrumentos y herramientas necesarias en la autogestión del aprendizaje, cognición orientada a la autonomía, y estrategias cognitivas.

En la continuidad de estos procesos de enseñanza y aprendizaje surgen otros elementos transversales al conectivismo y su relación con diferentes corrientes pedagógicas, a través de las cuales se visibiliza la conectividad continua, la movilidad como condición asincrónica para mantener esta conectividad, inmersos en el sistema y ecosistema, a través de una conexión consistente al interior de las redes, por medio de la cual, surge la ubicuidad en aprendizaje (Bair y Stafford,2016) comprende estar en varios sitios al mismo tiempo, posibilitando el acceso abierto, directo y continuo al aprendizaje; en particular, en apertura a las oportunidades para poder aprender.

Lo anteriormente mencionado se explica a su vez, tomando en cuenta los planteamientos de López-Gil (2016), quien propone las redes de aprendizaje y la internet como un contexto abierto a procesos constructivistas y colaborativos, basados en actividades informales. La movilidad en el aprendizaje a través de las herramientas digitales, permite configurar espacios colaborativos, en los cuales, las experiencias son compartidas, de manera formal e informal. Con este referente, la fluidez de información hace de las redes un espacio líquido (Bauman, 2006 apud López-Gil,2016) que se compila o contiene en ambientes virtuales específicos; pero a su vez, se transmite de manera continua, fluida, con capacidad para incidir en otras redes, tomar diferentes caminos en el sistema (rizoma), o integrarse a otros sistemas ya existentes a través de diversos puentes y medios de comunicación.

Siendo el conectivismo un paradigma pedagógico que asegura el aprendizaje en estos tiempos de rápido cambio y movimiento tecnológico (Foroughi et al.,2015); es a su vez, una tendencia pedagógica que aún se estudia en el contexto de la educación virtual por cuanto se propone comprender los fundamentos epistemológicos que dieron origen a este concepto, en especial, el procesamiento de información a gran escala para generar aprendizajes, los procesos de interacción e interactividad y, la relación continua entre los diferentes actores e individuos, docentes y estudiantes 
que hacen parte de una red educativa cualificada por la forma como internet y la Web (Web 1.0,2.0,3.0) se ha ido transformando hacia una fuente que compila gran cantidad de información y procesan rápidamente los datos (Foroughi et al., 2015).

Conforme una red, como un sistema o, como un ecosistema, el comportamiento de la red denota en lo pedagógico una dinámica basada en la cantidad de relaciones entre docentes, estudiantes y comunidades y, la información que allí se comparte. De igual forma, incide la calidad en la comunicación, en la permanencia, los lazos, uniones, contactos, nodos y demás, puntos de encuentro que mantienen el eje educativo de las relaciones entre quienes participan de los procesos educativos (Foroughi et al., 2015). Estos lazos, uniones, nodos de información y sistemas de distribución son condiciones de la perspectiva ecosistémica que se integra a una educación ambiental. Según Corrêa y Barbosa (2018), son características de la educación ambiental y la conciencia planetaria, la pluralidad del pensamiento, la transdisciplinariedad, la cooperación y responsabilidad, la sostenibilidad como actitud, la conciencia colectiva, el conocimiento pertinente, la incertidumbre y no certeza, la estructura social como sistema, y el holismo informático. De igual forma, la identidad personal compartida, la relación de la conciencia ética, los colectivos y el medio ambiente.

Con lo anterior, de acuerdo con Corrêa e Barbosa (2018) se propone el concepto conciencia activa, la cual comprende la conciencia colectiva ética, de carácter ambiental y sostenible. Aspectos que aunados al conectivismo permiten comprender las totalidades de la relación entre personas y los procesos de aprendizaje para promover la digitación, la red y las nuevas conexiones que atienden a una mirada ecológica, ambiental y sostenible, en el marco de una pedagogía que se orienta hacia la integración cognitiva, conductual, constructivista y conectivista para una sociedad educativa en red.

\section{EL CONECTIVISMO EN LA DINÁMICA REDES DE APRENDIZAJE Y COMUNIDADES DE PRÁCTICA}

La condición de red como sistema, y ecosistema, se integra a las prácticas que se desarrollan con base en los procesos cognitivos y de construcción del conocimiento caracterizados fundamentalmente por la relación red aprendizaje, procesos de pensamiento y la cognición como proceso de orden motivacional y afectivo. De igual forma, la generación de los procesos neurocognitivos se fundamenta en el desarrollo de habilidades mentales para integrar y fortalecer redes en interacción en conexión continua.

De acuerdo con Cao (2018), la exposición exponencial a un gran cúmulo de información exige del estudiante una disposición abierta al procesamiento cognitivo continuo, que se integra continuamente a los procesos de pensamiento y lenguaje. De igual forma, requiere de una construcción continua, una aplicación en contexto y una discusión permanente con las comunidades. Como en los ecosistemas, las redes neuronales de aprendizaje se caracterizan por promover procesos de pensamiento en donde la cognición determina aspectos reflexivos, analíticos, interpretativos y 
prácticos, que procuran buscar continuas conexiones y fortalecer las relaciones. En este sentido, la configuración de relaciones implica además, tomar decisiones y establecer contactos, la continua conexión para actualizar procesos, el mantener una esperanza en los aprendizajes como algo posible y viable; son a su vez, la habilidad para construir conocimiento, las redes o caminos que se identifican para fortalecer tal proceso y, la habilidad para conectar fuentes de información o nodos que compilan información y, que se desarrollan conjuntamente para generar lazos de comunicación entre diversas fuentes y repositorios.

Siguiendo a Dag (2017), la conformación de redes de aprendizaje basadas en el conectivismo conllevan a la creación de ambientes interconectados y distribuidos, en donde el mismo estudiante puede autodirigir su proceso y generar o integrar experiencias, a partir de aquí, el mismo estudiante puede generar nuevas conexiones y relaciones, desarrolla habilidades de autoorganización y mantenimiento de las relaciones y contactos para fortalecer el sistema y, promover un proceso de similaridad con la figura del rizoma. Sobre este modelo de conexión comunicacional la realimentación (metacognición) cumple un papel fundamental caracterizado primordialmente por el flujo de información pertinente que permite ajustar, adaptar y reacomodar el aprendizaje alcanzado en un continuo sin cierre absoluto del proceso educativo.

Con lo anterior, en las investigaciones de Melo Fiallos et al.(2017) se analizan las funcionalidades de las redes a partir de los principios conectivistas, cognitivos y socio-constructivos en ambientes educativos virtuales, como un flujo de información que se distribuye como un conocimiento abierto; basado en la configuración ambientes educativos con un origen autodirigido, según las necesidades de aprendizaje del individuo y las conexiones generadas a través de este mismo. En estos contextos tecnológicos, las experiencias educativas en especial, con pedagogías conectivistas virtuales, se profundizan a través de una construcción continua y expansiva del conocimiento. Por medio de la cual, se hace pertinente desarrollar experiencias que comprendan conocimientos aplicados y discusiones abiertas sobre los saberes reflexivos y bajo un punto central de encuentro (Dobozy, 2015). Según Yumurtaci (2017) y Goldie (2016), la condición expansiva del conocimiento, encuentra su justificación en la red basada en procesos conectivistas, cognitivos y socio-constructivos, a través de una dinámica constante y móvil que está sustentada en el acceso, la permanencia y, la fluidez de la comunicación, los datos, la información distribuida en redes que se extienden a lo largo de los canales de información y los actores red inmersos en todo el sistema, esta información es cambiante, dinámica, integrada a otras redes, en continua construcción entre la comunidad y el individuo.

No obstante, y con base en lo planteado por Bolívar y Dávila (2016), las buenas prácticas en los contextos de la educación virtual requieren de un continuo procesamiento de la información por parte del individuo y la comunidad, en donde la planeación instruccional, la gestión en la apertura y cierre de cursos y demás periodos de formación continua, están determinados por métodos de control, seguimiento, realimentación y evaluación de los procesos de aprendizaje que son procesados por la administración educativa y la orientación del docente hacia el logro de los procesos educativos propios de las carreras o programas formativos. 
Con un nivel de autorregulación y control de los procesos de aprendizaje, gestión y evaluación de la información y de la apropiación teórico-práctica, se configuran comunidades como espacios colectivos caracterizados por estrategias sociales de construcción del conocimiento (Pérez, 2015; Saritas, 2015). Por medio de las cuales se crean alianzas y compromisos, siendo estas condiciones psicológicas un factor comportamental que se encadena a los procesos cognitivos y neuronales de cada individuo, quien configura su propia red en relación con el espacio virtual y la colectividad. Siendo una red colectiva, teje conocimientos y saberes con sentido cohesionado y participativo. Entre el conjunto de redes, existen aquellas que mantienen mayor grado de cohesión y por ende, determina ciertos tipos de comportamientos asociados al compromiso y la filiación por un ideal. Estas comprenden, interacción, relación, experiencias de diálogo compartidas o, transmisión de ideales comunes.

Las comunidades de práctica según Ravenscroft (2011), se caracterizan por un diálogo abierto por medio del cual se permite explorar los logros de aprendizaje obtenidos a través de un grupo de participantes, los mecanismos de regulación adoptados por la comunidad posibilitan identificar experiencias y categorías novedosas para un nuevo nivel de aprendizaje, el fortalecimiento de los procesos de aprendizaje y estructuras cognitivas. Los niveles de aprendizaje son un camino para generar nuevas conexiones, relaciones e interacciones entre los medios tecnológicos, los agentes mediadores y demás individuos que conforman una comunidad. La práctica se fortalece a partir de los niveles alcanzados, las experiencias alcanzadas por un grupo, las acciones compartidas que se repiten, reproducen y transmiten a otras comunidades.

El medio más eficiente para fortalecer una red en comunidad es el diálogo continuo y el lenguaje. Estos son necesarios para mantener una red a través de experiencias verbales, que son fuente de sinergias integradas a la perspectiva ecológica del pensamiento y su configuración como un ecosistema en continuo aprendizaje (Boitshwarelo, 2011). Esta condición conlleva a la cohesión entre las experiencias e ideas compartidas, para lo anterior, el diálogo se configura como una necesidad continua que debe practicarse a diario. La existencia ecosistémica de las redes depende del diálogo entre individuos, y el buen uso del lenguaje; es decir, su diversificación orientada a generar espacios de discusión, problematización, comprensión de diferentes realidades o puntos de vista y de la relación cada vez más estrecha entre individuo, consigo mismo, con el aprendizaje alcanzado, su relación con el medio ambiente, las ecologías del pensamiento y las tecnologías.

Lo anterior se relaciona con los planteamientos de Aguilar y Mosquera (2015), para quienes la ecología del conocimiento se desarrolla a través de momentos pedagógicos, cada uno de los cuales se sitúan en los entornos de aprendizaje, referenciados desde las etapas de construcción de una red como parte de una sucesión ecológica en donde la biología de los ecosistemas se integran a conocimientos situados en las redes virtuales y sistemas de distribución del conocimiento, al cual se le denomina eco-conectivismo. De esta forma, los ambientes de aprendizaje a través de la digitalidad son sensibles al contexto (Aguilar y Mosquera, 2015), la 
gestión y uso continuo de las tecnologías, los entornos virtuales de aprendizaje se inspiran en el concepto de "sucesión ecológica", por el cual se plantean procesos evolutivos y auto-adaptativos de entorno personales de aprendizaje o entornos personalizados en conectividad (Muni y Rabell, 2017). La visión ecológica aquí presente se comprende en el sentido de poder establecer vínculos entre los seres vivos y el entorno que los rodea.

Finalmente, las sinergias que mantienen cohesionada una red, comprenden una geografía del conectivismo y las comunidades inmersas en la misma (Sinka, 2011). Estas condiciones implican el uso de tecnologías comunicacionales que necesariamente se vinculan al medio ambiente como un ecosistema en continuo cambio, dentro del cual, existen responsabilidades compartidas entre quienes conforman una red generando vínculos continuos y dinámicos con el medio ambiente. Esta relación medio ambiente y comunicación se visibiliza a través de las redes, las cuales se fortalecen por medio de una homeóstasis social y se construyen elementos de interconexión de orden humano-tecnológica. Según Sinka (2011), la competencia de la sociedad de la información es un estado maduro de información en la sociedad, que puede convertirse en foco de nuevos espacios de investigación en el futuro para generar relaciones e interacciones con mayor sentido en la condición de una red ecosistémica basada en ecologías del aprendizaje y del pensamiento socialmente distribuido.

\section{APUNTES FINALES}

Los análisis desarrollados anteriormente demuestran que el conectivismo se inspira e inspira las relaciones entre individuos, comunidades, información, conocimiento y ecosistemas. Cada uno de estos se interrelacionan de tal forma, que su funcionamiento determina orden, caos y complejidad; esto es, su condición para ser una tendencia pedagógica contemporánea se sustenta en la comprensión de los demás conceptos como aprendizajes emergentes, ecologías de aprendizaje, ecosistemas de aprendizaje, actor red, redes de aprendizaje, aprendizaje rizoma, aprendizaje nómada, comunidades de práctica. Estas categorías determinan una mirada alterna al proceso de aprender, la cual no se sustenta precisamente como lo plantea el conectivismo, en el aprendizaje como una experiencia que no reside en el individuo, sino, como algo que existe en la red; condición que actualmente se critica desde los abordajes del aprendizaje a través de una visión crítica del conectivismo (Zapata-Ros, 2015).

Lejos de anular lo que los grandes teóricos del aprendizaje ya han puntuado, el conectivismo refuerza la complejidad del conocimiento, lo que nos permite vincular las tesis de la complejidad a la metacognición; o sea, el conocimiento que las personas tienen sobre sus propios procesos cognitivos y la habilidad de controlar estos y poder desarrollarlos. En el conectivismo el conocimiento no es un producto sino proceso que se distribuye a través de una red de conexiones, por lo tanto, el aprendizaje consiste en la capacidad de construir y atravesar redes, donde los elementos que participan del sistema (personas o signos) contribuyen a la ramificación de las "nuevas" conexiones mentales. 
En la actualidad se ha moldeado una nueva visión del conocimiento y del aprendizaje y cada vez más queda evidente que la adquisición del conocimiento no ocurre exclusivamente por vías institucionales, conforme a la perspectiva tradicional de educación inmersa en el aula. Finalmente, tomando en cuenta los aportes de Siemens (2005); los procesos educativos y tecnológicos han incidido en la forma como se configuran las relaciones humanas, la forma como se vive y como se comunica el ser humano ha determinado que el aprendizaje se acompañe hoy en día de procesos novedosos en la integración tecnología, digitalidad y prácticas educativas orientadas hacia la formación en nuevos conocimientos.

\section{REFERENCIAS}

AGUILAR,J.; MOSQUERA, D. Middleware reflexivo para la gestión de aprendizajes conectivistas en ecologías de conocimientos (eco-conectivismo). Latin American Journal of Computing, Quito, v. 2, n. 2, p. 25-31, 2015. Disponible en: https://lajc. epn.edu.ec/index.php/LAJC/article/view/87/49. Acceso en: 2 oct. 2018. https://doi. org/10.13140/RG.2.1.3813.6402

ALDAHDOUH, A. A. Jumping from one resource to another: how do students navigate learning networks? International Journal of Education al Technology in Higher Education, Sweden, v. 15, n. 45, 2018. Disponible en: https:// educationaltechnologyjournal.springeropen.com/articles/10.1186/s41239-018-0126-x. Acceso en: 25 oct. 2018. https://doi.org/10.1186/s41239-018-0126-x

BAIR, R. A.; STAFFORD, T. Connected and ubiquitous: a discussion of two theories that impact the future learning applications. Techtrends - Linking Research and Practice to Improve Learning, Bloomington, v. 60, n. 2, p. 129-135, Mar. 2016. Disponible en: https://eric.ed.gov/?id=EJ1094177. Acceso en: 2 dic. 2018. https://doi. org/10.1007/s11528-016-0021-z

BAGGALEY, J. Thesis and antithesis. Distance Education, London, v. 33, n. 1, p. 117-123, 2012. Disponible en: https://sci-hub.tw/https://doi.org/10.1080 /01587919.2012.667963. Acceso en: 2 dic. 2018. https://doi.org/10.1080/0158 7919.2012.667963

BAUMAN,Z. Modernidad líquida. Buenos Aires: Fondo de Cultura Económica, 2006. BENEDETTI, F. Designing an effective and scientifically grounded e-learning enviroment for initial teacher education: the Italian University line model. Journal of E-Learning and Knowledge Society, Reggio Emilia, v. 14, n. 2, p. 97-109, 2018. Disponible en: https://www.learntechlib.org/p/184463/.Acceso en: 2 dic. 2018. https:// doi.org/10.20368/1971-8829/1510

BOITSHWARELO, B. Proposing an integrated research framework for connectivism: utilising theoretical synergies. International Review of Research in Open and Distance Learning, Athabasca, v. 12, n. 3, p. 161-179, 2011. Disponible en: http:// www.irrodl.org/index.php/irrodl/article/view/881. Acceso en: 2 dic. 2018. https://doi. org/10.19173/irrodl.v12i3.881 
BOLÍVAR, C. R.; DÁVILA, A. A. Propuesta de buenas prácticas de educación virtual en el contexto universitario. RED - Revista de Educación a Distancia, Murcia, v. 49, n. 12, 2016. Disponible en: https://www.um.es/ead/red/49/bolivar_davila.pdf. Acceso en: 2 dic. 2018. http://dx.doi.org/10.6018/red/49/12

BORNA, M.; FOULADCHANG, M. The motivational outcomes of connectivism theory in EFL. Modern Journal of Language Teaching Methods, Iran, v. 8, n. 2, p. 101-112, 2018. Disponible en: http://mjltm.org/browse.php?a_id=278\&slc_ lang=en\&sid=1\&printcase $=1 \&$ hbnr=1\&hmb=1. Acceso en: 25 oct. 2018 . https://doi. org $/ 10.26655 / \mathrm{mjltm} .2018 .2 .8$

BOZKURT,A.;HONEYCHURCH,S.;CAINES,A.;BALI,M.;KOHTROPOULOS, A.; CORMIER, D. Community tracking in a cMOOC and nomadic learner behavios identificationon a connectivit rhizomatic learning network. Turkish Online Journal of Distance Education, Turkey,v. 17, n. 4, p. 4-30,2016. Disponible en: https://core.ac.uk/ download/pdf/46664079.pdf. Acceso en: 25 nov. 2018. http://dx.doi.org/10.17718/ tojde.09231

BOZKURT, A.; KEEFER,J.Participatory learning culture and community formation in connectivist MOOCs. Interactive Learning Enviroments, United Kingdom, v. 26, n. 6, p. 776-788, 2018. Disponible en: https://www.tandfonline.com/doi/abs/10.1080 /10494820.2017.1412988. Acceso en: 25 nov. 2018. https://doi.org/10.1080/104948 20.2017.1412988

BROWN, J. S. Learning, working \& playing in the digital age. In: National Teaching and Learning Forum. Conference on Higher Education. Washington, DC: AAHE, 1999. s/p.

CAO, L. Y. Study of College English teaching interaction and teaching practice base on connectivism from the neurocognitive perspective. Educational Science Theory and Practice, Brisbane, v. 18, n. 5, p. 2.338-2.346, 2018. Disponible en: https://jestp. com/index.php/estp/article/view/320/286. Acceso en: 28 marzo 2020. https://doi. org/10.12738/estp.2018.5.132

CCI - Centro Cochrane Iberoamericano. Manual Cochrane de revisiones sistemáticas de intervenciones: versión 5.1.0. Barcelona: Cochrane Iberoamericano, 2011. Disponible en: https://community.cochrane.org/book_pdf/224. Acceso en: 8 nov. 2017

CLARÀ, M.; BARBERÀ, E. Three problems with the connectivist conception of learning. Journal of Computer Assisted Learning, United States, v. 30, n. 3, p. 197206, 2014. Disponible en: https://onlinelibrary.wiley.com/doi/abs/10.1111/jcal.12040. Acceso en: 2 dic. 2017. https://doi.org/10.1111/jcal.12040

CLARK, K. R.; VEALÉ, B. L.; WATTS, L. K. A review of the use of Massive Open Online Courses (MOOCs) in medical imaging education. Internet Journal of Allied Health Science and Practice, United States, v. 15, n. 2, 2017. Disponible en: https:// nsuworks.nova.edu/ijahsp/vol15/iss2/1/. Acceso en: 2 dic. 2017.

CORRÊA, T.H. B.; BARBOSA, N. A.P.Educação ambiental e consciência planetária: uma necessidade formativa. Revista Eletrônica do Mestrado em Educação Ambiental, Rio Grande, v. 35, n. 2, p. 125-136, maio/ago. 2018. https://doi.org/10.14295/remea. 
v35i2.7676 CORREAA, T. H. B.; MORENO, C. P. C.; PINEDA, N. E. C. Reflexiones sobre el desarrollo del aprendizaje y la complejidad neurocognitiva. Quaestio - Revista de Estudos em Educação, Sorocaba, v. 20, n. 2, p. 455-470, 30 ago. 2018. https://doi. org/10.22483/2177-5796.2018v20n2p455-470

DAG, K. N. A scholar-practitioner perspectiveon a leadership development program in health care: integrating connectivism theory. Advances in Development Human Resources, Thousand Oaks, v. 19, n. 3, p. 295-313, 2017. Disponible en: https://journals. sagepub.com/doi/abs/10.1177/1523422317712671. Acceso en: 2 dic. 2018. https://doi. org/10.1177/1523422317712671

DELEUZE, G.; GUATTARI, F. Mil mesetas, capitalismo y esquizofrenia. Valencia: Pre-Textos, 1987.

DOBOZY, E. The learning management system discussion board as change agent. International Journal of Continuing Engineering Education and Life-long Learning, Switzerland, v. 25, n. 1, p. 103-115, 2015. Disponible en: https://www. inderscienceonline.com/doi/pdf/10.1504/IJCEELL.2015.066550. Acceso en: 2 dic. 2018. https://doi.org/10.1504/IJCEELL.2015.066550

DOWNES, S. New technology supporting informal learning. Journal of Emerging Technologies in Web Intelligence, United States, v. 2, n. 1, p. 27-33, 2010. Disponible en: http://www.jetwi.us/uploadfile/2014/1226/20141226041314947.pdf. Acceso en: 28 marzo 2020. https://doi.org/10.4304/jetwi.2.1.27-33

FALCÃO, E. V. A sociedade cibernética. CADERNOS EBAPE.BR, Rio de Janeiro, v. 4, n. 2, p. 1-10, jun. 2006. https://doi.org/10.1590/S1679-39512006000200008

MELO FIALLOS, D. F.; SILVA CHAVEZ, J. A.; INDACOCHEA MENDOZA, L. R.; NUNEZ CAMPANA, J. H. Technologies in higher education: public policies and social appropiation of their implementation. Revista Digital de Investigación en Docencia Universitaria, Lima, v. 11, n. 1, p. 193-206, 2017. Disponible en: http://dev.scielo.org.pe/scielo.php?script=sci_abstract\&pid=S222325162017000100013\&lng=en\&nrm=iso. Acceso en: 2 dic. 2018. http://dx.doi. org/10.19083/ridu.11.498

FOROUGHI, A.; YAN, G. J.; SHI, H.; CHONG, D. Z. Web 3.0 ontology based on similarity: a steptoward facilit ating learning in the Big Data age. Journal of Management Analytics, United Kingdom, v. 2, n. 3, p. 216-232, 2015. Disponible en: https://www.tandfonline.com/doi/abs/10.1080/23270012.2015.1067154. https://doi. org/10.1080/23270012.2015.1067154

GOLDIE, J. G. S. Connectivism: a know ledge learning theory for the digital age? Medical Teacher, United Kingdom, v. 38, n. 10, p. 1.064-1.069, 2016. Disponible en: https://www. tandfonline.com/doi/full/10.3109/0142159X.2016.1173661?scroll=top\&needAccess=true. Acceso en: 2 dic. 2018. https://doi.org/10.3109/0142159X.2016.1173661

HASSAN, S. S. S. Measuring attitude to wards learning science in Malaysan secondary school context: implications for teaching. International Journal of Science Education, United Kingdom, v. 40, n. 16, p. 2.044-2.059, 2018. Disponible en: https://www. tandfonline.com/doi/abs/10.1080/09500693.2018.1518614?journalCode=tsed20. Acceso en: 2 dic. 2018. https://doi.org/10.1080/09500693.2018.1518614 
HILL, S. B.; WILSON, S.; WATSON, K. Learning ecology - a new approach to learning and transforming ecological consciousness: experiences form social ecology in Australia. In: O'SULLIVAN, E. V.; TAYLOR, M. M. (eds.). Learning toward an ecological consciousness: selected transformative practices. New York: Palgrave MacMillan, 2004. p. 47-64. Disponible en: https://www.researchgate.net/ publication/315038305_Learning_Ecology_A_New_Approach_to_Learning_ and_Transforming_Ecological_Consciousness. Acceso: 26 mar. 2020. https://doi. org/10.1007/978-1-349-73178-7_4

JOKSIMOVIĆ, S.; DOWELL, N.; POQUET,O.; KOVANOVIĆ, V.; GAŠEVIĆ, D.; DAWSON,S.; GRAESSER,A.C. Exploring development of social capital in a CMOOC through language and discourse. Internet and Higher Education, Amsterdam, v. 36, p. 54-64, 2018. Disponible en: https://www.researchgate.net/publication/319987180_ Exploring_development_of_social_capital_in_a_CMOOC_through_language_and_ discourse. Acceso en: 2 dic. 2018. https://doi.org/10.1016/j.iheduc.2017.09.004

KIZITO, R. N. Connectivism in learning activity design: implications for pedagogicallybased technology adoption in African higher education contexts. International Review of Research in Open and Distance Learning, Athabasca, v. 17, n. 2, p. 19-39, 2016. Disponible en: https://files.eric.ed.gov/fulltext/EJ1093706.pdf. Acceso en: 2 dic. 2018. https://doi.org/10.19173/irrodl.v17i2.2217

LE GRANGE, L. The "theoretical foundations" of community service-learning: from taproots to rhizomes. Education as Change, United Kingdom, v. 11, n. 3, p. 3-13, 2007. https://doi.org/10.1080/16823200709487174

LÓPEZ-GIL, M. The learning-network: the internet as a learning context.Journal for Educators Teachers and Trainers, Granada,v. 7, n. 2,p. 142-154,2016. Disponible en: http://jett.labosfor.com/index.php/jett/article/view/221/297. Acceso en: 28 marzo 2020.

MABUAN, R. A. Confessions of a Moocer: an autoethnorafhic inquiry online distance education. Turkish Online Journal of Distance Education, Turkey, v. 19, n. 4, p. 198213, 2018. Disponible en: http://tojde.anadolu.edu.tr/yonetim/icerik/makaleler/1755published.pdf. Acceso en: 30 oct. 2018. https://doi.org/10.17718/tojde.471916

MATTAR,J. Constructivism and connectivism in education technology: active, situated, authentic, experiential, and anchored learning. RIED - Revista Iberoamericana de Educación a Distancia, Madrid, v. 21, n. 2, p. 201-217, 2018. Disponible en: http:// revistas.uned.es/index.php/ried/article/view/20055. Acceso en: 2 nov. 2018. http:// dx.doi.org/10.5944/ried.21.2.20055

MORENO, R.; MACEDO, M. Informações e poder na arena da internet. Revista de Informação e Sociedade, João Pessoa, v. 24, n. 1, p. 47-60, 2014. Disponible en: http:// www.periodicos.ufpb.br/ojs/index.php/ies/article/view/15252. Acceso en: 2 dic. 2018. MUNI,J.L.A.; RABELL, L. H. Connectivism: an alternative in professional training according to the development of the information and communications technologies. Dilemas Contemporáneos-Educación Políticas y Valores, Toluca, v. 4, n. 3, p. 1-15, 2017. Disponible en: http://files.dilemascontemporaneoseducacionpoliticayvalores. com/200003504-aae68abe19/17-5-35\%20Conectivismo.\%20Una\%20alternativa\%20 en.....pdf. Acceso en: 2 dic. 2018. 
PANDO, V. F. Teaching trends in virtual education. An interpretative approach. Propósitos y Representaciones, Lima, v. 6, n. 1, p. 463-505, 2018. Disponible en: https://files.eric.ed.gov/fulltext/EJ1182496.pdf. Acceso en: 2 nov. 2018. http://dx.doi. org/10.20511/pyr2018.v6n1.167

PÉREZ, M. A. G. Technological and psychological enrichment of the concept of communities of practice in distance education. RED - Revista de Educación a Distancia, Murcia, v. 47, n. 7, p. 1-22, 2015. Disponible en: https://digitum.um.es/ xmlui/bitstream/10201/47057/1/Technological\%20and\%20Psychologica1\%20 Enrichment $\% 20$ of $\% 20$ the $\% 20$ Concept $\% 20$ of $\% 20$ Communities $\% 20$ of $\% 20$ Practice\%20in\%20Distance\%20Education.pdf. Acceso en: 2 dic. 2018. http://dx.doi. org/10.6018/red/47/7

RAVENSCROFT, A. Dialogue and connectivism: a new approach to understanding and promoting dialogue-rich networked learning. International Review of Research in Open and Distance Learning, Athabasca v. 12, n. 3, p. 139-160, 2011. Disponible en: http://www.irrodl.org/index.php/irrod1/article/view/934/1676. Acceso en: 2 dic. 2018. http://dx.doi.org/10.19173/irrodl.v12i3.934

RODRÍGUEZ, A. J. R.; MOLERO, D. M. M. Conectivismo como gestión del conocimiento. RDHES - Revista Electrónica de Humanidades, Educación y Comunicación Social, Venezuela, año 4, n. 6, p. 73-85, 2009. Disponible en: https://dialnet.unirioja.es/servlet/articulo?codigo=2937200. Acceso en: 2 dic. 2018.

RODRÍGUEZ, K. L. F.; PEÑA, G. A.; LUZURIAGA, M. T. O.; CANO, W. N. S.; FRANCO, M. L. L. The guidin guide and its role in directing the teachinglearning process of the Ecuadorian Higher Education. Dilemas Contemporáneos: Educación, Política y Valores, Toluca, v. 5, n. 1, p. 1-27, 2017. Disponible en:http://files.dilemascontemporaneoseducacionpoliticayvalores.com/20000355583d9084d40/17-9-20.\%20La\%20gu\%C3\%ADa\%20orientadora\%2C\%20su\%20 rol\%20en\%201a\%20direcci\%C3\%B3n\%20del\%20trabajo\%20independiente....pdf. Acceso en: 2 dic. 2018.

SANTAELLA, L. Culturas e artes do pós-humano: da cultura das mídias à cibercultura. São Paulo: Paulus, 2003.

SARITAS, M. T. The emergent technological and theoretical paradigms in education: the interrelations of cloud computing (CC), connectivism and internet of things (IoT). Acta Polytechnica Hungarica, Hungary, v. 12, n. 6, p. 161-179, 2015. Disponible en: https://uni-obuda.hu/journal/Saritas_62.pdf. Acceso en: 5 dic. 2018. http://dx.doi. org/10.12700/APH.12.6.2015.6.10

SIEMENS, G. Connectivism: a learning theory for the digital age. International Journal of Instructional Technology \& Distance Learning, Ontario, v. 2, n. 1, p. 1-8, 2004. Disponible en: http://www.itdl.org/Journal/Jan_05/article01.htm. Acceso en: 28 marzo 2020.

SINKA, R. Geography of the connectivism. Információs Társadalom, Budapest, v. 11, n. 1, p. 136-154, 2011. Disponible en: https://www.researchgate.net/ publication/289164113_Geography_of_the_Connectivism. Acceso en: 2 dic. 2018. 
THOTA, N.; NEGREIROS,J. G.M. Introducing educational technologies to teachers: experience report. Journal of University Teaching and Learning Practice, Australia, v. 12,n.1, p.1-15,2015. Disponible en: https://ro.uow.edu.au/jutlp/vol12/iss1/5/ Acceso en: 5 dic. 2018.

TRNOVA, E.; TRNA, J. Motivational effect of communication technologies in connectivist science education. Online Journal of Communication and Media Technologies, United States, v. 5, n. 3, p. 107-119, 2015. Disponible en: https:// www.ojcmt.net/article/motivational-effect-of-communication-technologiesin-connectivist-science-education. Acceso en: 29 marzo 2020. https://doi. org/10.29333/ojcmt/2519

VAS, R.; WEBER, C.; GKOUMAS, D. Implementing connectivism by semantic technologies for self-directed learning. International Journal of Manpower, United Kingdom,v. 39, n. 8, p. 1.032-1.046, 2018. Disponible en: https://www.emeraldinsight. com/doi/abs/10.1108/IJM-10-2018-0330. Acceso en: 3 dic. 2018. https://doi. org/10.1108/IJM-10-2018-0330

WANG, Z.; CHEN, L.; ANDERSON, T. A framework for interaction and cognitive engagement in connectivist learning contexts. International Review of Research in Open and Distance Learning, Athabasca, v. 15, n. 2, p. 121-141, 2014. Disponible en: http://www.irrodl.org/index.php/irrodl/article/view/1709/2838. Acceso en: 2 dic. 2018. https://doi.org/10.19173/irrodl.v15i2.1709

WILLIAMS, R.; KAROUSOU, R.; MACKNESS, J. Emergent learning and learning ecologies in Web 2.0. International Review of Research in Open and Distance Learning, Athabasca, v. 12, n. 3, p. 39-59, 2011. Disponible en: http://www.irrodl.org/ index.php/irrod1/article/view/883. Acceso en: 2 dic. 2018. https://doi.org/10.19173/ irrodl.v12i3.883

YUMURTACI, O. A re-evaluation of mobile communication technology: a theoretical Approach for Technology Evaluation in Contemporary Digital Learning. Turkish Online Journal of Distance Education, Turkey, v. 18, n. 1, p. 213-223, 2017. Disponible en: https://eric.ed.gov/?id=EJ1124972. Acceso en: 2 dic. 2018.https://doi.org/10.17718/ tojde. 285817

ZAPATA-ROS, M. Teorías y modelos sobre el aprendizaje en entornos conectados y ubicuos. bases para un nuevo modelo teórico a partir de una visión crítica del "conectivismo". Education in the Knowledge Society, Salamanca, v. 16, n. 1, p. 69-102, 2015. Disponible en: https://www.researchgate.net/publication/235671949_Teorias_y_ modelos_sobre_el_aprendizaje_en_entornos_conectados_y_ubicuos_Bases_para_un_ nuevo_modelo_teorico_a_partir_de_una_vision_critica_del_conectivismo. Acceso en: 5 dic. 2018. https://doi.org/10.14201/eks201516169102

ZHOU, C. Y. Empirical study on the effectiveness of teaching model of college english writing wthin blended learning mode. Educational Science: Theory \& Practice, Australia, v. 18, n. 5, p. 1.060-1.076, 2018. Disponible en: https:// pdfs.semanticscholar.org/ecf8/3f2de99d85a07999e3b858bb5cdcf37a090e.pdf? $\mathrm{ga}=2.207526291 .1098354895 .1585406964-1963467456.1580396543$. Acceso en: 22 marzo 2020. https://doi.org/10.12738/estp.2018.5.009 


\section{SOBRE LOS AUTORES}

Jaime Andres Torres Ortiz es doctor en educación científica por la Universidad Pedagógica y Tecnológica de Colombia (Colombia). Profesor de la misma institución.

E-mail: jatomaster3@gmail.com

Thiago Henrique Barnabé Corrêa es doctor en quimica por la Universidade Estadual de Campinas (UNICAMP). Profesor de la Universidade Federal do Triângulo Mineiro (UFTM).

E-mail: correa.uftm@gmail.com

Recibido el 20 de julio de 2019

Aprobado el 10 de febrero de 2020 